\title{
Erythrodermia Congenitalis Ichthyosiformis Bullosa of Brocq
}

\author{
Dorothea Sander $^{\mathrm{a}} \quad$ Josef Schröder ${ }^{\mathrm{b}} \quad$ Ines Schönbuchner $^{\mathrm{c}} \quad$ Julia Schreml $^{\mathrm{d}}$ \\ Sigrid Karrer $^{a} \quad$ Mark Berneburg $^{a} \quad$ Stephan Schreml ${ }^{a}$ \\ ${ }^{a}$ Department of Dermatology, ${ }^{b}$ Central Electron Microscopy Lab at the Institute of \\ Pathology and 'Department of Human Genetics, University Medical Center Regensburg, \\ Regensburg, and ${ }^{d}$ Institute for Human Genetics, University of Cologne, Cologne, Germany
}

\section{Key Words}

Erythroderma $\cdot$ Ichthyosis $\cdot$ Keratin

\begin{abstract}
A 50-year-old man presented with congenital scaling and hyperkeratosis on his palms, the soles of his feet and the extensor areas of his joints. The flexural areas were unaffected. His maternal grandmother, questionably his maternal uncle, his mother, all three brothers, one of his two sisters as well as two nephews and three nieces have or had similar skin changes. A punch biopsy was taken from the left palm. Clinical and histological signs led to the diagnosis of erythrodermia congenitalis ichthyosiformis bullosa of Brocq. We confirmed this genetically and found a heterozygous duplication (c.1752dupT) in the keratin 1 gene (KRT-1). To our knowledge, this is the first case of this skin condition reported in the literature with a heterozygous duplication (c.1752dupT) in KRT-1.

\section{Case Report}

A 50-year-old man presented with congenital scaling and hyperkeratosis on his palms, the soles of his feet and the extensor areas of his joints. The flexural areas were unaffected. Emollients and clobetasol-containing ointments had only limited effect. The patient reported that his skin condition had significantly improved after he spent time at the Dead Sea. His maternal grandmother, questionably his maternal uncle, his mother, all three brothers, one of his two sisters as well as two nephews and three nieces have or had similar skin changes.

On examination, the palms showed significant hyperlinearity with strong desquamation, hyperkeratosis and fissures (fig. 1). The heels and soles exhibited marked fissures and

\section{KARGER}

Stephan Schreml, MD, PhD

Department of Dermatology, University Medical Center Regensburg

Franz-Josef-Strauss-Allee 11

DE-93053 Regensburg (Germany)

E-Mail stephan@schreml.de 
hyperkeratosis (fig. 1d). Furthermore, there was hyperpigmentation on the inner thighs (fig. 1c), the buttocks, the palms and the extensor surfaces of the metacarpophalangeal joints.

A punch biopsy taken from the left palm showed orthohyperkeratosis, which was shifted upwards by a hyperparakeratotic layer. The epidermis exhibited psoriasiform akanthopapillomatosis, partly with hypergranulosis and partly with diminished stratum granulosum. Keratinocytes were strikingly pale and showed signs of disturbed maturation. There were also necrotic keratinocytes. In the upper dermis, discrete perivascular lymphocytic infiltrates were found (fig. 1e). Under electron microscopic analysis, intra- and extracellular lipid inclusions as well as segmental amyloid deposits in the upper dermis were detected (fig. 1f). The amyloid deposits were most likely secondary to chronic inflammation due to the epidermal barrier changes.

The clinical and histological signs led to the diagnosis of erythrodermia congenitalis ichthyosiformis bullosa of Brocq. We confirmed this genetically and found a heterozygous duplication (c.1752dupT) in the keratin 1 gene (KRT-1). This mutation has not been found in any other patient so far.

The hands and feet were treated with urea and salicylic acid-containing ointments, for the rest of the body ointments consisting of Vaseline and lanolin (unguentum molle) were used. The fissures were closed with superglue. Bath PUVA therapy was initiated for the hands and feet. Additionally, systemic treatment with acitretin $20 \mathrm{mg}$ once daily was started.

\section{Discussion}

Ichthyoses are genetic disorders of keratinization with often generalized, abnormally dry, scaly skin and hyperkeratosis. Ichthyoses can be divided into two main groups: noncongenital ichthyoses, which develop in the first weeks or months after birth (vulgar ichthyoses), and congenital ichthyoses, which are clinically seen at the day of birth [1-3]. Erythrodermia congenitalis ichthyosiformis bullosa of Brocq is a congenital ichthyosis caused by autosomal dominant and spontaneous mutations of keratin genes KRT-1 and KRT-10 [4]. These mutations lead to altered keratin intermediate filaments. The prevalence is estimated to be $1: 200,000[2,3]$. Clinically, this skin condition shows generalized erythema and erosions or blistering at the day of birth. Later, verrucous hyperkeratosis can often be found at the neck, the flexural areas as well as the palms and soles (especially in patients with KRT-1 mutations) [1-4]. To our knowledge, this is the first case of this skin condition reported in the literature with a heterozygous duplication (c.1752dupT) in KRT-1.

\section{Statement of Ethics}

We obtained written informed consent to publish the figures in this case.

\section{Disclosure Statement}

The authors have no conflict of interest. 
Sander et al.: Erythrodermia Congenitalis Ichthyosiformis Bullosa of Brocq

\section{References}

1 Bogenrieder T, Landthaler M, Stolz W: Bullous congenital ichthyosiform erythroderma: safe and effective topical treatment with calcipotriol ointment in a child. Acta Derm Venereol 2003;83:52-54.

-2 Ingen-Housz-Oro S, Vignon-Pennamen MD, Blanchet-Bardon C: Bullous and non-bullous ichthyosiform erythroderma associated with generalized pustular psoriasis of Zumbusch type. Br J Dermatol 2001;145: 823-825.

-3 Kucharekova M, Mosterd K, Winnepenninckx V, van Geel M, Sommer A, van Steensel MA: Bullous congenital ichthyosiform erythroderma of Brocq. Int J Dermatol 2007;46(suppl 3):36-38.

-4 Saeki H, Hattori N, Mitsui H, Adachi M, Imakado S, Ishibashi Y, Tamaki K: A keratin 10 gene mutation (Arg156Cys) in a Japanese patient with bullous congenital ichthyosiform erythroderma. J Dermatol 2002;29:168-171.
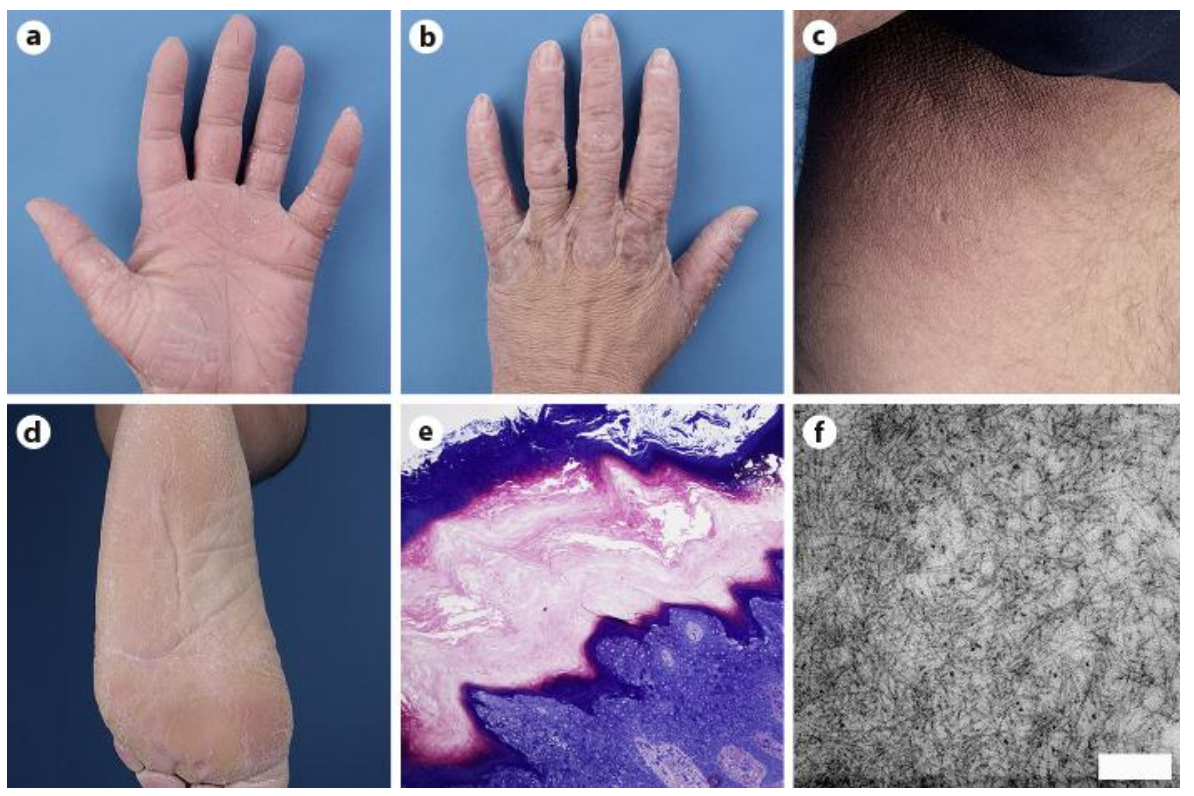

Fig. 1. Clinical images and microscopy. a, b Hands: significant hyperlinearity with desquamation as well as partial hyperkeratosis and fissures. c Inner thighs: hyperpigmentation. d Heels and soles of the feet: marked fissures and hyperkeratosis. e Histology with H\&E staining: orthohyperkeratosis shifted upwards by a hyperparakeratotic layer, psoriasiform akanthopapillomatosis, partly with hypergranulosis and partly with reduced stratum granulosum. Keratinocytes were strikingly pale and show signs of disturbed maturation. Necrotic keratinocytes were also found. Discrete perivascular lymphocytic infiltration subepidermally. f Electron microscopy: intra- and extracellular lipid inclusions (not shown) as well as segmental amyloid deposits (unbranched fibers in image) in the upper dermis (bar $=200 \mathrm{~nm}$ ). 